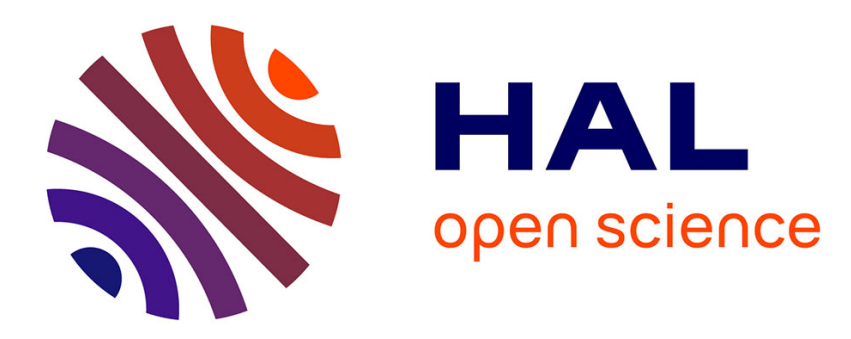

\title{
Capacity-approaching signals for the scalar Gaussian channel
}

\author{
Hugo Méric, Jérôme Lacan
}

\section{To cite this version:}

Hugo Méric, Jérôme Lacan. Capacity-approaching signals for the scalar Gaussian channel. Wireless Telecommunications Symposium 2016, Apr 2016, London, United Kingdom. pp. 1-3. hal-01434827

\section{HAL Id: hal-01434827 \\ https://hal.science/hal-01434827}

Submitted on 13 Jan 2017

HAL is a multi-disciplinary open access archive for the deposit and dissemination of scientific research documents, whether they are published or not. The documents may come from teaching and research institutions in France or abroad, or from public or private research centers.
L'archive ouverte pluridisciplinaire HAL, est destinée au dépôt et à la diffusion de documents scientifiques de niveau recherche, publiés ou non, émanant des établissements d'enseignement et de recherche français ou étrangers, des laboratoires publics ou privés. 


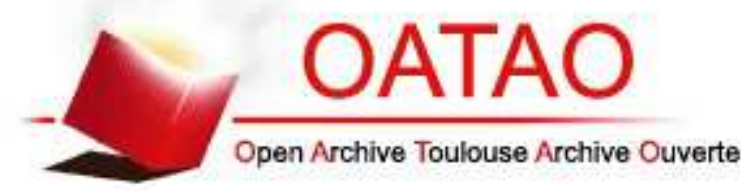

\section{Open Archive TOULOUSE Archive Ouverte (OATAO)}

OATAO is an open access repository that collects the work of Toulouse researchers and makes it freely available over the web where possible.

This is an author-deposited version published in: http://oatao.univ-toulouse.fr/ Eprints ID: 16227

To link this document: http://dx.doi.org/10.1109/WTS.2016.7482053

To cite this version: Méric, Hugo and Lacan, Jérôme Capacity-approaching signals for the scalar Gaussian channel. (2016) In: Wireless Telecommunications Symposium 2016, 18 April 2016 - 20 April 2016 (London, United Kingdom).

Any correspondence concerning this service should be sent to the repository administrator: staff-oatao@listes-diff.inp-toulouse.fr 


\section{Capacity-approaching signals for the scalar Gaussian channel}

\author{
Hugo Méric \\ NIC Chile Research Labs \\ Santiago, Chile \\ E-mail: hmeric@niclabs.cl
}

\author{
Jérôme Lacan \\ University of Toulouse \& ISAE/DISC \\ Toulouse, France \\ E-mail: jerome.lacan@isae.fr
}

\begin{abstract}
We consider the scalar Gaussian channel with power constraint $P$. A gap exists between the channel capacity and the highest achievable rate of equiprobable uniformly spaced signal. Several approaches enable to overcome this limitation such as constellations with non-uniform probability or constellation shaping. In this short paper, we focus on constellation shaping. We present a method to construct one-dimensional constellations with equiprobable signaling that achieve the Gaussian capacity as the number of signal points goes to infinity.
\end{abstract}

\section{INTRODUCTION}

We consider the scalar additive white Gaussian noise (AWGN) channel with signal-to-noise ratio (SNR) $P / N_{0}$, where $P$ is the average power constraint of the input signal and $N_{0}$ the noise variance. Let $\mathrm{snr}=P / N_{0}$, the Gaussian channel capacity is

$$
C=\frac{1}{2} \log _{2}(1+\mathrm{snr}) \text { bits/dimension, }
$$

and the optimal input distribution is Gaussian with zero mean and variance $P[1]$.

Approaching the Gaussian capacity is a challenging problem in digital communications systems. Indeed the optimal input distribution is continuous while in practice the signal is chosen from a finite constellation of points. Several approaches enable to overcome this limitation such as constellation shaping or constellations with non-uniform probabilities [2]. In this letter, we focus on the former solution as it is more attractive for practical systems that mainly use equiprobable signaling.

Sun and van Tilborg were the first to present a sequence of random variables equiprobably distributed over a finite support that achieves the Gaussian capacity (in one dimension) as the signal set cardinality tends to infinity [3]. Until then, the traditional rule of designing constellations for the AWGN channel was to maximize the squared Euclidean distance between the signal points under the power constraint. In one dimension, this results in uniformly spaced signal sets that, when combined with equiprobable signaling, exhibit a gap of $\frac{\pi \mathrm{e}}{6} \approx 1.53 \mathrm{~dB}$ with the Gaussian capacity at large SNR [4]. Sun and van Tilborg shows how to close this gap in one dimension. In higher dimensions, this shaping gain can be obtained by choosing the signal points on an $N$-dimensional rectangular lattice from within an $N$-sphere rather than an $N$ cube [4]. However this is impractical when $N$ increases.

978-1-5090-0314-3/16/\$31.00 (c) 2016 IEEE
The result in [3] was extended by Schwarte that provided sufficient conditions for equiprobable input distributions with finite support to approach the Gaussian capacity in any dimension [5]. Similar conditions are given in [6, Theorem 9].

A recent work by $\mathrm{Wu}$ and Verdú studied the AWGN channel capacity with finite signal set [7]. They showed that as the input signal cardinality grows, the constellation capacity approaches the Gaussian capacity exponentially fast. They also introduced a family of constellation, based on the Hermite polynomials roots, achieving exponential convergence. The resulting modulations combine constellation shaping with nonuniform probabilities.

If the channel inputs are also subject to peak power constraints, the capacity and the optimal input distribution were studied for the scalar Gaussian channel in [8]. Smith showed that the capacity is achieved by a unique discrete random variable taking on a finite number of values. It was later extended to the quadrature Gaussian channel [9]. In two dimensions, the optimal distribution is discrete along the radial direction and continuous in the angular direction.

A very interesting paper by Huang and Meyn studies the structure of capacity-achieving input distributions [10]. One of the result is that discrete distribution can nearly reach the capacity even if the optimal distribution is continuous.

In this work, our main contribution (presented in Section II) is the construction of one dimensional constellations with equiprobable signaling that achieve the Gaussian capacity as the number of constellation points goes to infinity. To that end, we rely on a shaping function to produce signal sets with a Gaussian shape, the key ingredient to approach the AWGN channel capacity. This is an alternative to the previous solutions [3], [5]. We illustrate our result by evaluating the performance of a capacity-approaching constellation obtained from our proposal. We conclude the paper by discussing several research directions in Section III.

\section{CAPACITY-APPROACHING CONSTELLATIONS}

\section{A. Preliminary remarks}

Approaching the AWGN channel capacity with equiprobable Gaussian-shaped discrete distributions was first proposed by Sun and van Tilborg [3]. Based on this result, we recently introduced the concept of shaping function that aims to give a Gaussian shape to a finite constellation of points [11]. We 
proposed the Box-Muller transform as shaping function for the quadrature channel, resulting in capacity-achieving amplitude and phase-shift keying constellations [11].

Our current work focuses on the one-dimensional case and provides a general method to generate capacity-achieving signal sets with equiprobable signaling. Our choice of the shaping function relies on the following lemma:

Lemma 1. Let $U$ be the continuous uniform distribution in the interval $(0,1)$ and $\Phi_{P}$ the cumulative density function of the normal distribution with zero mean and variance $P$, then $\Phi_{P}^{-1}(U) \sim \mathcal{N}(0, P)$.

Based on the previous lemma, our idea is to consider $\Phi_{P}^{-1}$ as the shaping function for the one-dimensional case.

\section{B. Main result}

The following theorem explains how to generate capacityapproaching signal sets for the scalar AWGN channel. It is the main contribution of this letter.

Theorem. Let $\left(U_{n}\right)_{n \geqslant 1}$ be a sequence of discrete random variables that converge weakly to $U$, the continuous uniform distribution over $(0,1)$, such that $\sup _{n} \operatorname{Var}\left(\Phi_{P}^{-1}\left(U_{n}\right)\right)<\infty$. The signal defined by $X_{n}=\Phi_{P}^{-1}\left(U_{n}\right)$ achieves the AWGN channel capacity with power constraint $P$ as $n \rightarrow \infty$. More formally,

$$
I\left(X_{n} ; X_{n}+N\right) \underset{n \rightarrow \infty}{\longrightarrow} \frac{1}{2} \log _{2}(1+\mathrm{snr}),
$$

where I denotes the mutual information [1] and $N \sim$ $\mathcal{N}\left(0, N_{0}\right)$ is the Gaussian noise with variance $N_{0}$.

Proof: The idea is to show that the sequence $\left(X_{n}\right)_{n \geqslant 1}$ satisfies the two conditions given in [6, Theorem 9].

The first condition requires that $\left(X_{n}\right)_{n \geqslant 1}$ verifies $\sup _{n} \operatorname{Var}\left(X_{n}\right)<\infty$, which is true by hypothesis.

Next we prove that $\left(X_{n}\right)_{n \geqslant 1}$ converges weakly to the distribution of a Gaussian variable with zero mean and variance $P$. By assumption, we have that $U_{n} \stackrel{\mathcal{L}}{\rightarrow} U$ (weak convergence). The function $\Phi_{P}^{-1}$ is continuous on $(0,1)$, thus the continuous mapping theorem can be applied [12]. We obtain

$$
X_{n}=\Phi_{P}^{-1}\left(U_{n}\right) \stackrel{\mathcal{L}}{\rightarrow} \Phi_{P}^{-1}(U) .
$$

Lemma 1 ensures that $\Phi_{P}^{-1}(U) \sim \mathcal{N}(0, P)$.

Thus the signal defined by $X_{n}=\Phi_{P}^{-1}\left(U_{n}\right)$ satisfies both conditions in [6, Theorem 9]. We conclude that the signal $\left(X_{n}\right)_{n \geqslant 1}$ approaches the Gaussian channel capacity with power constraint $P$ as $n \rightarrow \infty$.

In the theorem, the assumption $\sup _{n} \operatorname{Var}\left(\Phi_{P}^{-1}\left(U_{n}\right)\right)<\infty$ is required to control the signal energy. This prevents to generate signals that approach the Gaussian distribution but may not have a finite variance. Another consequence is that $U_{n}$ can not take the values 0 or 1 , as in that case $\Phi_{P}^{-1}$ is not defined.

\section{Application}

To illustrate our result, we construct a capacity-achieving constellation with equiprobable signaling and evaluate its performance on the AWGN channel.

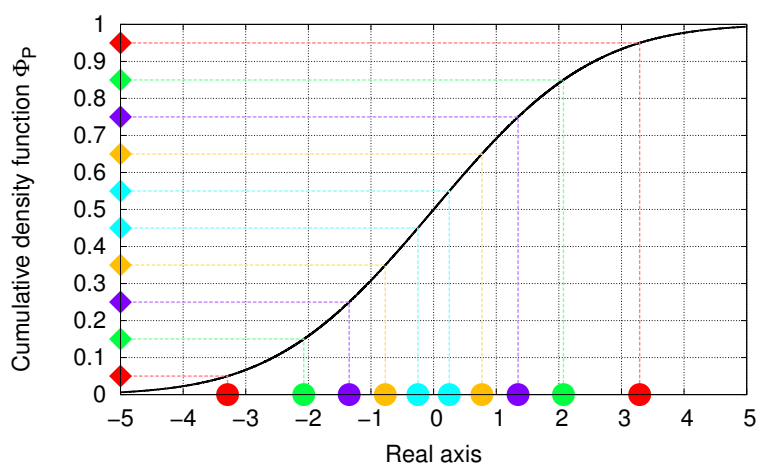

Fig. 1: Proposed Gaussian-shaped constellation for $n=10$ and $P=4$. On the vertical axis, the squares represent the support of $U_{n}$. On the real axis, the circles represent the support of $\Phi_{P}^{-1}\left(U_{n}\right)$, in other words the constellation $\mathcal{C}_{n}$.

Let $n \geqslant 1$ be an integer and $\mathbb{N}_{n}=\{0,1,2, \ldots, n-1\}$ the set of integers from 0 to $n-1$. We consider the discrete set

$$
\mathcal{S}_{n}=\left\{\frac{1}{2 n}+\frac{k}{n} \mid k \in \mathbb{N}_{n}\right\},
$$

that verifies $\left|\mathcal{S}_{n}\right|=n$ and $\mathcal{S}_{n} \subset(0,1)$. We introduce $U_{n}$ the discrete random variables uniformly distributed on $\mathcal{S}_{n}$ and the proposed signal is $X_{n}=\Phi_{P}^{-1}\left(U_{n}\right)$. As $U_{n}$ is uniformly distributed on $\mathcal{S}_{n}$, the random variable $X_{n}$ is uniformly distributed on a set $\mathcal{C}_{n}$ of $n$ points. The points in $\mathcal{C}_{n}$ exhibit a Gaussian shape as depicted in Fig. 1 for $n=10$ and $P=4$. This illustrates the objective of the shaping function: applying the shaping function to a set of uniformly spaced points generates a Gaussian-shaped constellation.

In order to show that the proposed constellations achieve the AWGN channel capacity, we prove that the two assumptions in the theorem are satisfied. The following lemma studies the convergence of $\left(U_{n}\right)_{n \geqslant 1}$.

Lemma 2. The sequence $\left(U_{n}\right)_{n \geqslant 1}$ converges weakly to $U$, the uniform distribution over $(0,1)$.

Proof: The characteristic function of $U_{n}$ is

$$
\begin{aligned}
\mathbb{E}\left[\mathrm{e}^{i t U_{n}}\right] & =\frac{1}{n} \sum_{k=0}^{n} \mathrm{e}^{i t\left(\frac{1}{2 n}+\frac{k}{n}\right)} \\
& =\frac{\mathrm{e}^{\frac{i t}{2 n}}}{n} \times \frac{1-\mathrm{e}^{i t}}{1-\mathrm{e}^{\frac{i t}{n}}} \underset{n \rightarrow \infty}{\longrightarrow} \frac{\mathrm{e}^{i t}-1}{i t}=\mathbb{E}\left[\mathrm{e}^{i t U}\right] .
\end{aligned}
$$

Using the continuity theorem [13, Theorem 26.3], we conclude that $\left(U_{n}\right)_{n \geqslant 1}$ converges weakly to $U$.

Next we study the variance of the sequence $\left(X_{n}\right)_{n \geqslant 1}$.

Lemma 3. $\left(X_{n}\right)_{n \geqslant 1}$ verifies $\sup _{n} \operatorname{Var}\left(X_{n}\right)<\infty$.

Proof: The variance of $X_{n}$ is equal to $\operatorname{Var}\left(X_{n}\right)=$ $\mathbb{E}\left[X_{n}^{2}\right]-\mathbb{E}\left[X_{n}\right]^{2}$. By construction of $\mathcal{S}_{n}, X_{n}$ is symmetrically distributed about the origin (see Fig. 1). Combined with the equiprobable signaling, this results in $\mathbb{E}\left[X_{n}\right]=0$.

Then we consider the term $\mathbb{E}\left[X_{n}^{2}\right]$ in the variance. First it is important to note that $\left(\Phi_{P}^{-1}\right)^{2}$ is Riemann integrable over 


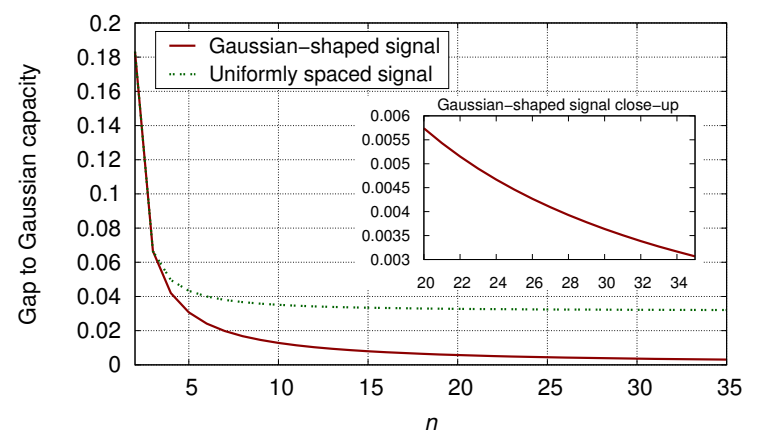

(a) $\mathrm{snr}=5 \mathrm{~dB}$

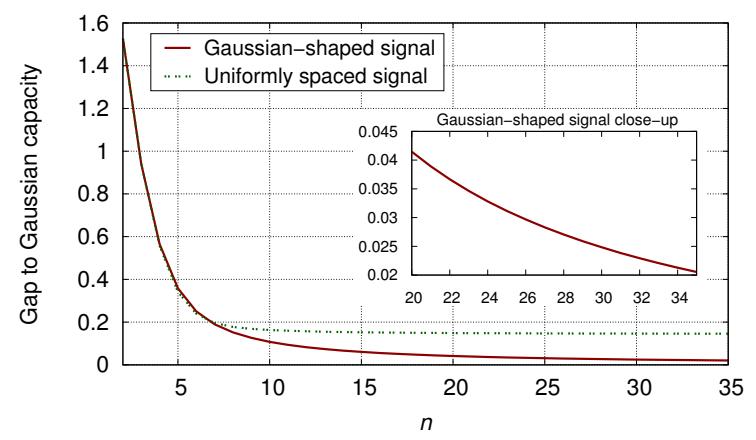

(b) $\mathrm{snr}=15 \mathrm{~dB}$

Fig. 2: Gap to the Gaussian capacity for the uniformly spaced signal with equiprobable signaling and the proposed constellation

$(0,1)$. Indeed, we have

$$
\int_{0}^{1} \Phi_{P}^{-1}(t)^{2} \mathrm{~d} t=\int_{\mathbb{R}} u^{2} \Phi_{P}^{\prime}(u) \mathrm{d} u=P,
$$

where we use the substitution $u=\Phi_{P}^{-1}(t)$. By definition,

$$
\mathbb{E}\left[X_{n}^{2}\right]=\sum_{k=0}^{n-1} \frac{1}{n} \Phi_{P}^{-1}\left(\frac{1}{2 n}+\frac{k}{n}\right)^{2} .
$$

The right term in (8) is a Riemann sum, therefore

$$
\mathbb{E}\left[X_{n}^{2}\right] \underset{n \rightarrow \infty}{\longrightarrow} \int_{0}^{1} \Phi_{P}^{-1}(t)^{2}=P .
$$

This concludes the proof that $\sup _{n} \operatorname{Var}\left(X_{n}\right)<\infty$.

Previous lemmas show that the sequence $\left(U_{n}\right)_{n \geqslant 1}$ satisfies both assumptions in the theorem. Thus, the Gaussianshaped signal with equiprobable signaling $X_{n}$ asymptotically approaches the AWGN channel capacity. Fig. 2 depicts the gap to Gaussian capacity for the uniformly spaced signal with equiprobable signaling and the proposed constellation for various SNR values and $2 \leqslant n \leqslant 35$. As expected, the capacity gap vanishes for the proposed constellation as $n \rightarrow \infty$. However the convergence speed remains an open question.

\section{CONCLUDING REMARKS}

Based on a shaping function, we introduce a constellation design enabling to achieve the Gaussian capacity as the signal cardinality grows to infinity. In that sense, the proposed constellations are better than uniformly spaced signals with equiprobable signaling. We illustrate our result by presenting and evaluating the performance of a sequence of capacityapproaching signals. It is important to note that the design is not unique as modifying the set $\mathcal{S}_{n}$, with respect to the assumptions given in the theorem, may generate other constellations that asymptotically achieve the Gaussian capacity. Our last remark is to point out that our solution can produce capacity-approaching constellations in higher dimensions. For instance in two dimensions, we can take two capacityachieving signals (for the one-dimensional case) transmitted on two quadrature carriers.
Future work will focus on three main directions. First, we plan to study the convergence speed of the proposed constellations. For the scalar AWGN channel with input cardinality $n$, Wu and Verdú showed that the achievable rate approaches exponentially fast the Gaussian capacity as $n$ grows [7]. However their solution combine constellation shaping and non-uniform probabilities, while we restrict our work to constellation shaping which is more attractive from a practical point of view. Then, we will seek the optimal constellations in terms of capacity (for a given SNR) or convergence speed. Finally, we will investigate if the representation of the input signal $X_{n}$ using $U_{n}$ may accelerate the decoding process.

\section{REFERENCES}

[1] T. Cover and J. Thomas, Elements of Information Theory. John Wiley \& sons, 2006.

[2] F. Kschischang and S. Pasupathy, "Optimal nonuniform signaling for Gaussian channels," Information Theory, IEEE Transactions on, vol. 39, no. 3, pp. 913-929, 1993.

[3] F.-W. Sun and H. van Tilborg, "Approaching capacity by equiprobable signaling on the Gaussian channel," Information Theory, IEEE Transactions on, vol. 39, no. 5, pp. 1714-1716, 1993.

[4] G. Forney, R. Gallager, G. Lang, F. Longstaff, and S. Qureshi, "Efficient modulation for band-limited channels," Selected Areas in Communications, IEEE Journal on, vol. 2, no. 5, pp. 632-647, 1984.

[5] H. Schwarte, "Approaching capacity of a continuous channel by discrete input distributions," Information Theory, IEEE Transactions on, vol. 42, no. 2, pp. 671-675, 1996.

[6] Y. Wu and S. Verdú, "Functional properties of MMSE," in Information Theory Proceedings, IEEE International Symposium on, 2010, pp. 14531457.

[7] — "The impact of constellation cardinality on Gaussian channel capacity," in Communication, Control, and Computing, 48th Annual Allerton Conference on, 2010, pp. 620-628.

[8] J. Smith, "The information capacity of amplitude- and varianceconstrained scalar Gaussian channels," Information and Control, vol. 18, no. 3, pp. 203-219, 1971.

[9] S. Shamai and I. Bar-David, "The capacity of average and peakpower-limited quadrature Gaussian channels," Information Theory, IEEE Transactions on, vol. 41, no. 4, pp. 1060-1071, 1995.

[10] J. Huang and S. Meyn, "Characterization and computation of optimal distributions for channel coding," Information Theory, IEEE Transactions on, vol. 51, no. 7, pp. 2336-2351, 2005.

[11] H. Méric, "Approaching the Gaussian channel capacity with APSK constellations," Communications Letters, IEEE, vol. 19, no. 7, pp. 11251128 , July 2015.

[12] P. Billingsley, Convergence of probability measures (Second edition). Wiley.

[13] - Probability and measure (Third edition). Wiley. 